\title{
ASYMPTOTIC DIOPHANTINE APPROXIMATIONS AND EQUIVALENT NUMBERS ${ }^{1}$
}

WILLIAM W. ADAMS

Let $\alpha$ be a real irrational number. Define for all real numbers $B \geqq 1$, $\lambda(B, \alpha)$ to be the number of solutions in integers $p, q$ satisfying the inequalities

$$
|q \alpha-p|<1 / q \text { and } 1 \leqq q \leqq B .
$$

The purpose of this note is to consider the following question. If $\alpha$ and $\alpha^{\prime}$ are equivalent irrational numbers (see [2] for all terminology) then is

$$
\lambda(B, \alpha) \sim \lambda\left(B, \alpha^{\prime}\right) ?
$$

We show that this is usually the case. But there are exceptions, namely when the partial quotients of $\alpha$ grow too fast.

Let $\alpha=\left[a_{0}, a_{1}, a_{2}, \ldots\right]$ be expressed by means of its continued fraction. Let $\alpha_{n}=\left[a_{n}, a_{n+1}, \cdots\right]$ and $p_{n} / q_{n}=\left[a_{0}, a_{1}, \cdots, a_{n}\right]$. From [1] we have the following formula for counting:

Lemma. Set for all $\nu \geqq 0, \lambda_{\nu}=\left[\left(\alpha_{\nu+1}+q_{\nu-1} / q_{\nu}\right)^{1 / 2}\right]+\rho_{\nu}$ where $\rho_{\nu}$ is computed as follows:

if $a_{\nu+1}=1$ then $\rho_{\nu}=0$,

if $a_{\nu+1}=2$ then $\rho_{\nu}=1$,

if $a_{\nu+1} \geqq 3$ then $\rho_{\nu}$ has a contribution of 1 for the truth of each of the following:

$$
\begin{aligned}
\alpha_{\nu+1} & <q_{\nu} / q_{\nu-1}+2, \\
q_{\nu+1} / q_{\nu} & <\alpha_{\nu+2}+2,
\end{aligned}
$$

(so $\left.\rho_{\nu}=0,1,2\right)$. Then given any $k, 1 \leqq k \leqq\left[\left(\alpha_{n+1}+q_{n-1} / q_{n}\right)^{1 / 2}\right]$, we have

$$
\lambda\left(k q_{n}, \alpha\right)=\sum_{\nu=0}^{n-1} \lambda_{\imath}+k+O(1) \quad(n \rightarrow \infty) .
$$

$([x] \equiv$ largest integer $\leqq x)$.

The main result is expressed in terms of continued fractions. In corollaries we express results for $\lambda(B, \alpha)$.

Theorem. Let $\alpha=\left[a_{0}, a_{1}, \cdots\right]$ and $\alpha^{\prime}=\left[a_{0}^{\prime}, a_{1}^{\prime}, \cdots\right]$ be equiva-

Received by the editors December 2, 1966.

1 This research was partially supported by National Science Foundation grant GP-3990 and a National Science Foundation postdoctoral fellowship. 
lent irrational numbers; so say that for $n \geqq n_{0}, a_{n+l+1}=a_{n}{ }^{\prime}$. Let $\lambda_{\nu}, \rho_{\nu}$ for $\alpha$ and $\lambda_{\nu}^{\prime}, \rho_{\nu}^{\prime}$ for $\alpha^{\prime}$ be the quantities of the lemma. Let $n_{0}<n_{1}<n_{2}<\cdots$ be the integers such that $\lambda_{n_{\nu}+l+1} \neq \lambda_{n_{\nu}}^{\prime}, \quad \nu=1,2,3, \cdots$.

Then there is a constant $C>1$ such that $n_{\nu}>C^{\nu}, \quad \nu=1,2,3, \cdots$.

Proof. It clearly suffices to assume that $\alpha=\left[a_{0}, \cdots, a_{l}, \alpha^{\prime}\right]$. So suppose $n>n_{0}$ is such that $\lambda_{n+l+1} \neq \lambda_{n}{ }^{\prime}$. Then certainly

$$
\left[\left(\alpha_{n+l+2}+q_{n+l} / q_{n+l+1}\right)^{1 / 2}\right] \neq\left[\left(\alpha_{n+1}^{\prime}+q_{n-1}^{\prime} / q_{n}^{\prime}\right)^{1 / 2}\right]
$$

or the inequality in (1) or in (2) are different for primed and unprimed symbols. It suffices to consider all the possible cases separately and show that the indices where the given case has inequality increases exponentially. The same type of argument works in all the cases so we assume, say, that

$$
\left[\left(\alpha_{n+l+2}+q_{n+l} / q_{n+l+1}\right)^{1 / 2}\right]>\left[\left(\alpha_{n+1}^{\prime}+q_{n-1}^{\prime} / q_{n}^{\prime}\right)^{1 / 2}\right]
$$

and show these indices increase exponentially.

Now since $a_{n+l+1}=a_{n}^{\prime}$ for all $n \geqq 0$ we have also $\alpha_{n+l+1}=\alpha_{n}{ }^{\prime}$. Then in order for (3) to hold we must have $a_{n+l+2}=a_{n+1}^{\prime}=a^{2}-1$ for some in teger $a \geqq 2$. Then (3) is equivalent to

$$
\alpha_{n+l+3}^{-1}+q_{n+l} / q_{n+l+1}>1>\alpha_{n+2}^{\prime-1}+q_{n-1}^{\prime} / q_{n}^{\prime} .
$$

In particular we have $q_{n+l} / q_{n+l+1}>q_{n-1}^{\prime} / q_{n}^{\prime}$ which is equivalent to $n$ being even since $q_{l+2} / q_{l+1}>q_{1}^{\prime} / q_{0}^{\prime}$. Further, for the left inequality in (4) to hold, we must have either $a_{n+l+1}=1$ or $a_{n+l+3}=1$. Again it suffices to show separately that the indices $n$ where $a_{n+l+3}=1$ and (3) holds and where $a_{n+l+1}=1$ and (3) holds increase exponentially. Again the arguments are essentially the same so we assume that $a_{n+l+3}=1$. Then one checks easily that (4) is equivalent to

$$
q_{n}^{\prime} / q_{n-1}^{\prime}>1+\alpha_{n+3}^{\prime}=1+\alpha_{n+l+4}>q_{n+l+1} / q_{n+l} .
$$

So we see that

$$
a_{n+l+1}=1+a_{n+l+4}
$$

and

$$
q_{n-1}^{\prime} / q_{n-2}^{\prime}<\alpha_{n+4}^{\prime}=\alpha_{n+l+5}<q_{n+l} / q_{n+l-1} .
$$

Hence $a_{n+l}=a_{n+l+5}$. Continue and we see that for all $g, \quad 0 \leqq g \leqq n-2$, we have

$$
a_{n+l-o}=a_{n+l+5+o}
$$

and 


$$
\alpha_{2 n+l+4}>q_{l+1} / q_{l}
$$

(using the fact that $n$ is even). To recapitulate we say the sequence of partial quotients of $\alpha$ has property $P_{n}$ for an integer $n$ provided $n$ is even, $a_{n+l+2}=a^{2}-1, \quad a_{n+l+3}=1$ and (5), (6) hold. We will show that the sequence of integers $n$ such that $P_{n}$ is true increases exponentially.

Suppose that $P_{n}, P_{n+2 r}, P_{n+2 s}, 0<r<s$ are true. We will show that $s \geqq n / 10$. So suppose to the contrary that $s<n / 10$.

Now let $t=r$ or $s$. Then for $1 \leqq g \leqq n-2$ we see that

$$
a_{n+l+4+g}=a_{n+l+4+g+4 t}
$$

by using $P_{n}$ and $P_{n+4 t}$, that is the sequence $a_{n+l+5}, \cdots, a_{2 n+l+2}$ is periodic of periods $4 r$ and $4 s$. Also the remainder of the sequence mod $4 t$ starts the next period. Continue the sequence to be an infinite sequence of period $4 s$. Then the resulting sequence has period $4 r$ also; that is for all integers $x \geqq 0$

$$
a_{n+l+5+x}=a_{n+l+5+x+4 r} .
$$

To see this we note that it is clear when $n+l+5+x+4 r \leqq 2 n+l+2$, that is when $x \leqq n-3-4 r$. Since $0<r<s$ and $10 s<n$ we see that $4 s \leqq n-3-4 r$. Thus writing $x=4 s q+p, \quad 0 \leqq p<4 s$ we have

$$
a_{n+l+5+x}=a_{n+l+5+p}=a_{n+l+5+p+4 r}=a_{n+l+5+x+4 r},
$$

as desired.

So let $\mu$ be the minimum period of this sequence. Then $\mu \mid 4 r$ and $\mu \mid 4 s$. Since $r<s$ we have $\mu<4 s$. Singling out some sequence of $4 s$ terms we have the following type of sequence

$$
\begin{array}{llllllllllllll}
b_{1} & b_{2} & b_{3} & b_{4} & \cdots & b_{2 s-1} & b_{2 s} & b_{2 s+1} & b_{2 s+2} & b_{2 s+3} & b_{2 s+4} & \cdots & b_{4 s-1} & b_{4 s} \\
x & 1 & y & v_{4} & \cdots & v_{28-1} & z & 1 & i & j & v_{2 s-1} & \cdots & v_{4} & u
\end{array}
$$

where $x \neq 1$, which has period $\mu \mid 4 s, \mu<4 s$. We show that this is impossible, giving the desired contradiction.

Write $4 s=\rho \mu$ where $\rho>1$. First note that $2 \nmid \rho$, since if it did then $2 s=\rho^{\prime} \mu$ and

$$
x=b_{1}=b_{1+\rho^{\prime \mu}}=b_{1+2 s}=1 .
$$

Thus in particular $\mu \geqq 4$ and $\rho \geqq 3$. Now for all $g$ satisfying $2 \leqq g \leqq 2 s-3$ we have $b_{2 s+1-g}=b_{2 s+2+g}$. Choose integers $\eta$, $\zeta$ satisfying $1 \leqq \eta<\zeta$ and $\eta+\zeta=\rho$. Then one can check that

$$
2 s+4 \leqq 2+\zeta \mu \leqq 4 s-1, \quad 1 \leqq 1+\eta \mu \leqq 4 s .
$$


Thus letting $g=2+\zeta \mu-(2 s+2)=\zeta \mu-4 s$ we have

$$
\begin{aligned}
1 & =b_{2+\zeta \mu}=b_{2 s+2+\rho}=b_{2 s+1-(\zeta \mu-2 s)} \\
& =b_{4 s+1-\zeta \mu}=b_{1+\mu(\rho-\zeta)}=b_{1}=x
\end{aligned}
$$

a contradiction.

The contradiction was obtained by assuming $s<n / 10$ so that $s \geqq n / 10$. Suppose we have $P_{n_{1}}, P_{n_{2}}, P_{n_{3}}, \cdots$. Then with $n=n_{v}$ $n+2 r=n_{\nu+1}, n+2 s=n_{\nu+2}$ we have

$$
n_{p+2}-n_{\nu} \geqq n_{\nu} / 5 \text { or } n_{\nu+2} \geqq 6 n_{v} / 5 \text {. }
$$

Thus we see $n_{\nu} \geqq C^{v}$ for some $C>1$ as desired. This completes the proof of the theorem.

The notation will continue to be the same as that set up in the theorem.

CoRollary 1. For all $n$ we have with $1 \leqq k \leqq\left[\left(\alpha_{n+1}+q_{n-1} / q_{n}\right)^{1 / 2}\right]$, $\lambda\left(k q_{n}^{\prime}, \alpha^{\prime}\right)=\lambda\left(k q_{n+l+1}, \alpha\right)+O\left(\log \log q_{n}^{\prime}\right)(n \rightarrow \infty)$.

Proof. Since $\left|\lambda_{n+l+1}-\lambda_{n}{ }^{\prime}\right| \leqq 3$ for any $n$ we have from the theorem and lemma

$$
\begin{aligned}
\lambda\left(k q_{n}^{\prime}, \alpha^{\prime}\right) & =\sum_{\nu=0}^{n-1} \lambda_{\nu}^{\prime}+k+O(1)=\sum_{\nu=l+1}^{n+l} \lambda_{\nu}+k+O(\log n) \\
& =\sum_{\nu=0}^{n+l} \lambda_{\nu}+k+O(\log n)=\lambda\left(k q_{n+l+1}, \alpha\right)+O(\log n)
\end{aligned}
$$

and so the result follows since $n=O\left(\log q_{n}{ }^{\prime}\right)$.

The next corollary says, in particular, that for almost all numbers $\alpha, \alpha$ has the same asymptotic estimate as any number equivalent to $\alpha$ (see [3]).

Corollary 2. If $\lambda(B, \alpha) \sim C \log B$ for some constant $C>0$, and $\alpha^{\prime}$ is equivalent to $\alpha$, then $\lambda(B, \alpha) \sim \lambda\left(B, \alpha^{\prime}\right)$.

Proof. There is a constant $C_{1}>0$ such that $q_{n}{ }^{\prime} \leqq q_{n+l+1} \leqq C_{1} q_{n}^{\prime}$ and so from Corollary 1 we see that $\lambda\left(k q_{n}{ }^{\prime}, \alpha\right) \sim C \log k q_{n}^{\prime}$ $\left(1 \leqq k \leqq\left[\left(\alpha_{n+1}+q_{n-1} / q_{n}\right)^{1 / 2}\right]\right)$. Then given $B$ there is an $n$ and such a $k$ with $k q_{n}^{\prime} \leqq B<(k+1) q_{n}^{\prime}$ or else $\left[\left(\alpha_{n+1}+q_{n-1} / q_{n}\right)^{1 / 2}\right] q_{n}^{\prime} \leqq B<q_{n+1}^{\prime}$; say $N_{1} \leqq B<N_{2}$. Then since $\lambda\left(N_{1}, \alpha^{\prime}\right)=\lambda\left(N_{2}, \alpha^{\prime}\right)+O(1)$ we are done.

Of course, if an explicit error term had been given we could derive one for the difference between $\lambda(B, \alpha)$ and $\lambda\left(B, \alpha^{\prime}\right)$.

CoRollary 3. There are equivalent numbers $\alpha$ and $\alpha^{\prime}$ such that $\lambda(B, \alpha)$ and $\lambda\left(B, \alpha^{\prime}\right)$ are not asymptotic. 
Proof. It is clear that if $l \geqq 2$ and $\alpha=\left[a_{0}, \cdots, a_{l}, \alpha^{\prime}\right]$ then there is a $C_{1}>1$ (depending only on $a_{0}, \cdots, a_{l}$ ) such that $q_{n+l+1} \geqq C_{1} q_{n}^{\prime}$. So given $a_{0}, \cdots, a_{l}, l \geqq 2$ we now construct $\alpha^{\prime}$. Note first from the Lemma

$$
\lambda\left(q_{n}^{\prime}, \alpha^{\prime}\right)=\sum_{\nu=1}^{n} a_{\nu}^{\prime 1 / 2}+O(n) .
$$

So we may define inductively integers $k_{n}, a_{n+1}$ such that

$$
\lim _{n \rightarrow \infty} \frac{1}{k_{n}} \lambda\left(q_{n}^{\prime}, \alpha^{\prime}\right)=0 \text { and } 1 \leqq C_{1} k_{n}<a_{n+1}^{\prime 1 / 2}
$$

Then using Corollary 1 we see that

$$
\lim _{n \rightarrow \infty} \frac{\lambda\left(k_{n} q_{n+l+1}, \alpha\right)}{\lambda\left(k_{n} q_{n+l+1}, \alpha^{\prime}\right)} \leqq \lim _{n \rightarrow \infty} \frac{\lambda\left(k_{n} q_{n}^{\prime}, \alpha^{\prime}\right)}{\lambda\left(C_{1} k_{n} q_{n}^{\prime}, \alpha^{\prime}\right)}=\frac{1}{C_{1}}<1
$$

as" desired.

However we have the following

CoRollary 4. Let $\alpha, \alpha^{\prime}$ be any two equivalent irrationals. Then

$$
\lambda\left(q_{n}, \alpha\right)=\lambda\left(q_{n}, \alpha^{\prime}\right)+O\left(\log \log q_{n}\right) \quad(n \rightarrow \infty)
$$

(the error term is smaller than the main term by [1]).

Proof. Since $q_{n}^{\prime} \leqq q_{n+l+1} \leqq C_{2} q_{n}^{\prime} \quad\left(C_{2}>0\right.$, constant $)$ and $\lambda\left(k q_{n}^{\prime}, \alpha^{\prime}\right)$ $\leqq \lambda\left(q_{n}^{\prime}, \alpha^{\prime}\right)+k+O(1)$ for any $k$ we have by Corollary 1

$$
\begin{aligned}
\lambda\left(q_{n+l+1}, \alpha\right) & =\lambda\left(q_{n}^{\prime}, \alpha^{\prime}\right)+O\left(\log \log q_{n}^{\prime}\right) \\
& =\lambda\left(q_{n+l+1}, \alpha^{\prime}\right)+O\left(\log \log q_{n+l+1}\right) .
\end{aligned}
$$

\section{REFERENCES}

1. W. Adams, $A$ lower bound in asymptotic diophantine approximations, Duke Math. J. 35 (1968).

2. S. Lang, Introduction to diophantine approximations, Addison-Wesley, Reading, Mass., 1966.

3. W. Schmidt, $A$ metrical theorem in Diophantine approximations, Canad. J. Math. 11 (1960), 619-631.

University of California, Berkeley and

Institute for Advanced Study, Princeton 